\title{
A malaria transmission-directed model of mosquito life cycle and ecology
}

\author{
Philip A Eckhoff
}

\begin{abstract}
Background: Malaria is a major public health issue in much of the world, and the mosquito vectors which drive transmission are key targets for interventions. Mathematical models for planning malaria eradication benefit from detailed representations of local mosquito populations, their natural dynamics and their response to campaign pressures.
\end{abstract}

Methods: A new model is presented for mosquito population dynamics, effects of weather, and impacts of multiple simultaneous interventions. This model is then embedded in a large-scale individual-based simulation and results for local elimination of malaria are discussed. Mosquito population behaviours, such as anthropophily and indoor feeding, are included to study their effect upon the efficacy of vector control-based elimination campaigns.

Results: Results for vector control tools, such as bed nets, indoor spraying, larval control and space spraying, both alone and in combination, are displayed for a single-location simulation with vector species and seasonality characteristic of central Tanzania, varying baseline transmission intensity and vector bionomics. The sensitivities to habitat type, anthropophily, indoor feeding, and baseline transmission intensity are explored.

Conclusions: The ability to model a spectrum of local vector species with different ecologies and behaviours allows local customization of packages of interventions and exploration of the effect of proposed new tools.

\section{Background}

Malaria is transmitted by the blood feeding of infectious female Anopheles mosquitoes, and understanding mosquito ecology and population dynamics can inform how best to defeat malaria. Malaria is an important global health issue, causing over half a billion cases and on the order of one million deaths a year [1], and is the focus of a global eradication campaign announced in 2007. Basic vector ecology is a fundamental driver of transmission patterns, and changes in land usage [2] or land modification can dramatically change transmission for better or worse. The growing urbanization in Africa is a powerful current example of such phenomena [3]. Climate and weather affect larval development and parasite maturation within the infected mosquito, and spatial models are able to predict malaria prevalence based primarily on climate details in the absence of interventions [4]. This climate-driven predictability has broken down more recently, possibly due to more widespread

Correspondence: peckhoff@intven.com

Intellectual Ventures Laboratory, $1600132^{\text {nd }}$ Ave NE, Bellevue, WA 98004 USA

\section{() Biomed Central}

interventions such as insecticide-treated bed nets [5], but predictive modelling for global eradication incorporates these geographic effects on baseline transmission. Geographic variation and spatial effects become increasingly important as heterogeneity in transmission allows malaria to persist in some areas while other areas achieve elimination but remain at risk of reintroduction [6].

A successful global eradication campaign will include substantial vector control components, and mathematical models for planning eradication will benefit from accurate and robust representation of the basic vector transmission ecology in each area of interest as well as the ability to incorporate interventions both singly and in combination. Vector population dynamics exhibit latencies such as the time required for sporogony. Spatial processes include vector oviposition, larval habitat, host-seeking, and migration. For aptly modelling eradication, representation of the steady state is not sufficient; elimination-predictive models may need to be accurate at very low prevalence. Finally, models must address sensitivity of results to model parameters and 
assumptions whenever presenting possible routes to eradication.

Mathematical modelling of the vector-borne transmission of malaria dates back to the early dynamical models of Ross and Macdonald [7,8], the classical assumptions of which have been clearly exposited [9]. Next steps in vector modelling included cyclical feeding models, which were easier to parameterize from field data and more accurately tracked the mosquito life cycle $[10,11]$. Other models emphasize the effect of rainfall and temperature correlations to transmission [12], or compute the Entomological Inoculation Rate (EIR) driven by human infectiousness [13]. Recent work has focused on the effect of hydrology on larval habitat and vector prevalence [14], and on vector population dynamics [15]. Other groups have built comprehensive simulations for both the vector transmission dynamics and withinhuman parasite dynamics [16-19]. Vector population models have been constructed for other vector-borne diseases, such as dengue [20].

The present work introduces a vector model which has detailed vector population resolution for near elimination phenomena, tracks explicit latencies of larval development and sporogony, implements closed-loop population dynamics, and can implement a wide variety of vector control interventions in combination. Careful attention is given to vector behaviours, such as host preference and feeding locations, and the effects of these parameters on intervention effectiveness are explored. Alternative implementations of this model are discussed, along with parameter sensitivities. The present model is then exercised on several issues of local elimination for simulations based on transmission patterns for a singlelocation in Tanzania, varying baseline transmission and vector bionomics, and key results are discussed.

\section{Methods: model design} Aquatic habitat

Available larval habitat is a primary driver of local mosquito populations, and different mosquito species can have different habitat preferences, with utilization of an ecological niche driving speciation in some cases [21]. Classifications of larval habitat include temporary, permanent or semi-permanent [22], and some species, such as Anopheles gambiae ss and Anopheles arabiensis will share an ecological niche for larval habitat [23]. Humans can affect available habitat through terrain changes which affect hydrology, through agricultural practices, such as rice cultivation [2], or through creating or eliminating standing water. Remote sensing through satellite imagery is becoming a powerful tool for mapping vector ecology $[2,23]$, and this trend will most likely continue to increase as eradication planning drives increasing data requirements. Several detailed models already exist of habitat and the impact of rainfall, temperature, humidity, and soil quality [14,24,25].

Rainfall and humidity can strongly affect available larval habitat $[14,22,23]$, although this depends on the mosquito species and its habitat preference. In fact, preference can be more specific than the species level, as Anopheles funestus exhibits differences in population responses to rainfall which are correlated with chromosomal diversity [26]. Rainfall, rather than habitats with water, is best correlated with numbers of An. gambiae s.l. This effect is not as strong as it is for culicines, nor is it universal, since An. gambiae s.l. have been found in stable aquatic habitats [22]. Even An. funestus, which prefers more semi-permanent larval habitat, has a rainfall dependence in its larval habitat [27], partly due to vegetation on edges of water [26] and the interaction of rainfall with agricultural schedules for crops such as rice.

In the present model, different models for larval habitat are developed for temporary, semi-permanent, permanent, and human-driven habitats. Temporary habitat $\mathrm{H}_{\text {temp }}$ in a grid of diameter $\mathrm{D}_{\text {cell }}$ increases with rainfall $\mathrm{P}_{\text {rain }}$ and decays with a rate $\tau_{\text {temp }}$ proportional to the evaporation rate driven by temperature $\mathrm{T}(\mathrm{K})$ and humidity RH:

$$
\begin{aligned}
& H_{\text {temp }}+=P_{\text {rain }} K_{\text {temp }} D_{\text {cell }}^{2}-H_{\text {temp }}\left(\frac{\Delta t}{\tau_{\text {temp }}}\right) \\
& \frac{1}{\tau_{\text {temp }}}=\left(5.1 \times 10^{11} \mathrm{~Pa}\right) e^{\frac{-5628.1 \mathrm{~K}}{T_{K}}} k_{\text {tempdecay }} \sqrt{\frac{.018 \mathrm{~kg} / \mathrm{mol}}{2 \pi R T_{K}}}(1-R H)
\end{aligned}
$$

in which the exponential results from the ClausiusClayperon relation, the root is from the expression for vapour evaporation rates due to molecular mass given a partial pressure, and the constant is the Clausius-Clayperon integration constant multiplied by a factor $\mathrm{k}_{\text {temp- }}$ decay to relate mass evaporation per unit area to habitat loss. The value of $\mathrm{k}_{\text {tempdecay }}$ is initially chosen to set the habitat half-lives near 1 day for hot and dry conditions and 2-3 weeks for more typical tropical conditions. The variation in $\tau_{\text {temp }}$ with temperature $\mathrm{T}$ and humidity $\mathrm{RH}$ can be seen in Figure 1a. Semi-permanent habitat increases with a constant $\mathrm{K}_{\text {semi }} \mathrm{D}_{\text {cell }}{ }^{2} \mathrm{P}_{\text {rain }}$ and decays with a longer time constant $\tau_{\text {semi. }}$. Permanent habitat is fixed at $\mathrm{K}_{\text {perm }} \mathrm{D}_{\text {cell }}{ }^{2}$, and human population-driven habitat is calculated as population $\mathrm{N}^{*} \mathrm{~K}_{\text {pop. }}$. The values of $k_{\text {tempdecay }}, \tau_{\text {semi }}, K_{\text {semi }}$, and $K_{\text {temp }}$ can be fit to local data on vector abundance by species over time or to local data on EIR to tailor a simulation to a specific setting.

Larval development and mortality rates are affected by a variety of factors including weather and densities of other larvae. Climate and weather affect not only larval habitat availability but also larval development rates and 

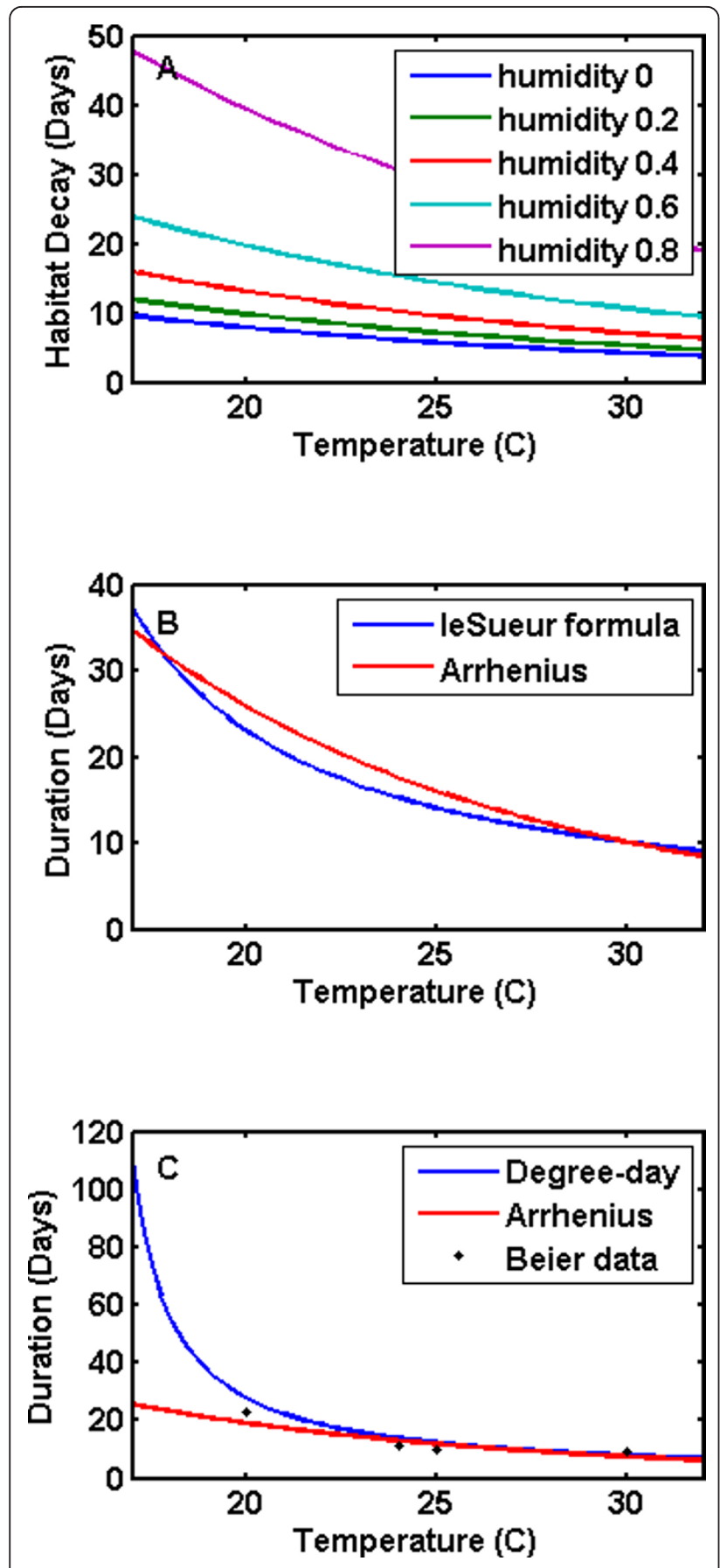

Figure 1 Effects of climate and weather on vector populations. a) Effect of temperature and humidity on time constant $\tau_{\text {temp }}$ for temporary rainfall-driven larval habitat. The habitat decay is faster for warmer and drier weather. b) Temperature effects on duration larval development, with the functional form from [4] and the present Arrhenius formulation. c) Temperature effects on duration of sporogony. The traditional degree-day formula and the present Arrhenius function are plotted, along with Beier's data from [49]. larval mortality [15]. The duration of larval development is a decreasing function of temperature [4], and the present model replaces earlier mathematical formulations [4] with an Arrhenius temperature-dependent rate $a_{1} \exp$ $\left(\mathrm{a}_{2} / \mathrm{T}_{\mathrm{K}}\right)$ as seen in Figure 1b. In some cases, this temperature-dependent rate must be modified by local larval density [28], although the presented results do not include such a modification. Rainfall and temperature then combine through habitat creation and larval development to create varying local patterns of distribution by larval instar [23], and larval mortality and development duration determine pupal rates [29].

Heavy rainfall can directly kill larvae by dislodging them from habitat and causing them to dry out [28]. Other factors increasing mortality are cannibalism of $1^{\text {st }}$ instar larvae by $4^{\text {th }}$ instar larvae and overpopulation of larval habitat acting to reduce food availability.

The present model includes this preferential survival of older larvae during overpopulation conditions by only treating as viable those new larvae, which do not cause the larval population to exceed capacity. If capacity shrinks so that the existing population exceeds available capacity, mortality is increased by the degree of overpopulation. With all these factors taken together, about 28 percent of larvae typically survive egg to adult [28]. The present model includes a daily larval mortality, which translates into a probability of survival of larval development as a function of temperature and mortality rate presented in Figure 2a. The larval survival plotted is from egg hatch to emergence, not from oviposition to adult maturity, which is significantly less due to egg survival and death during the immature phase.

\section{Improved cohort model}

There are different possible implementations of the basic model, each with different computational efficiencies, resolutions, and flexibilities. Possible implementations include a modified cohort simulation, a cohort simulation with explicit mosquito ages, a simulation of every individual mosquito in the population, as well as a simulation of a sampled subset of mosquitoes to represent the population as the whole. The basic model is presented in the context of the modified cohort simulation with explanations of the modifications for individual mosquitoes. In the modified cohort simulation, rather than representing the entire population by three compartments for susceptible, latently infected, and infectious mosquitoes, the simulation dynamically allocates a cohort for every distinct state, and the cohort maintains the count of all mosquitoes in that state. This allows temperature-dependent 

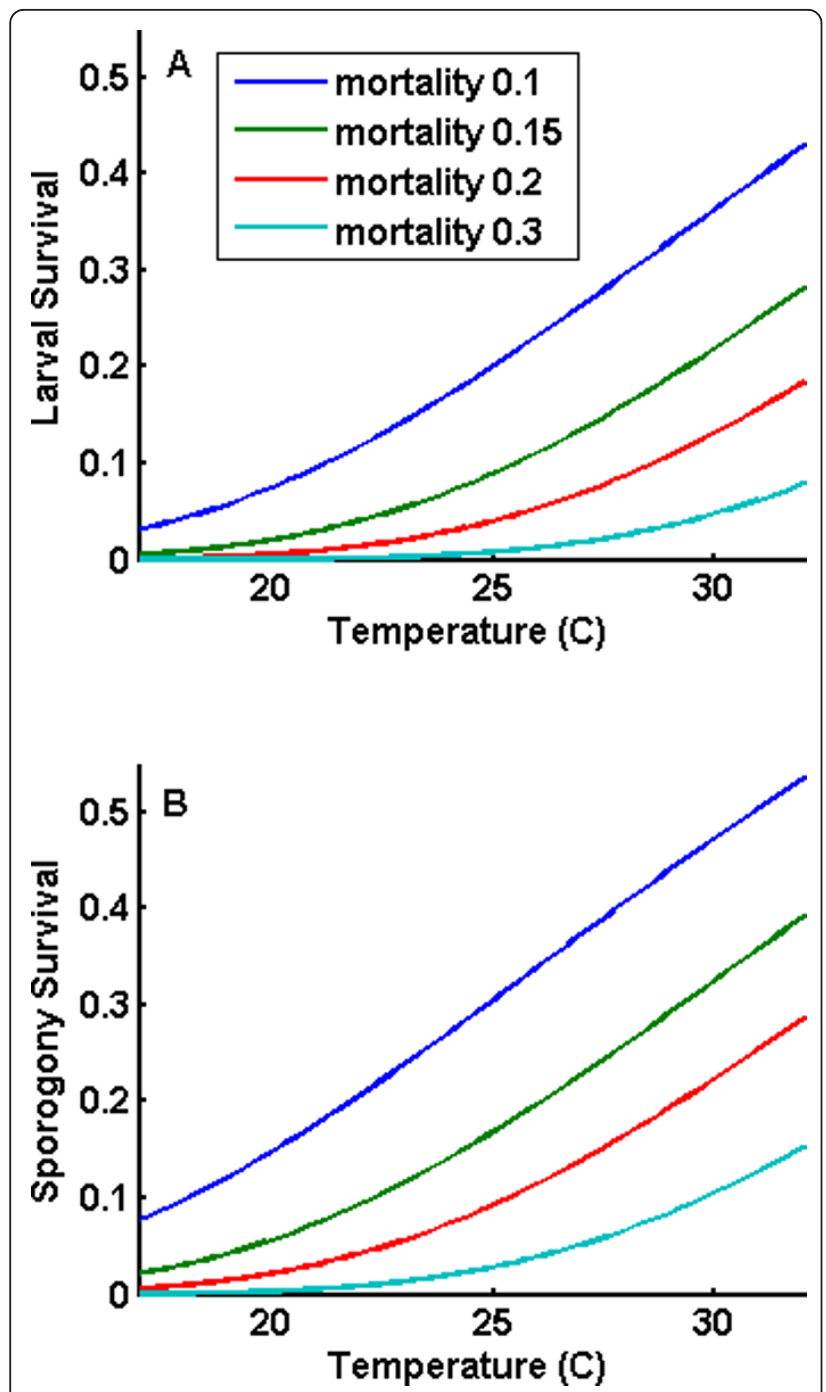

Figure 2 Intermediate outputs which affect vector population or disease transmission dynamics. a) Larval survival of development as a function of temperature and larval mortality. Survival is from successful egg hatch to emergence; survival from oviposition would be significantly lower. b) Adult survival of sporogony as a function of temperature and adult mortality. At lower temperatures, mosquitoes spend longer in each progress queue and the overall effect of a daily mortality is greater. Adult mortality can be artificially increased through interventions such as bed nets, insecticide spraying, and baited traps.

progression through sporogony as described below, even with a different mean temperature each day, with no mosquitoes passing from susceptible to infectious before the full discrete latency. For the cohort simulation with explicit ages to allow modelling of senescence, mosquito age is part of the state definition, and many more cohorts are required to represent the population. The overall progression of cohorts or individual mosquitoes through different states is outlined in Figure 3.

\section{Immature mosquito populations}

Upon emergence, there is a period of hours to days before bloodmeal-seeking begins [30]. This period is represented in the model as a fixed latency, during which predators and interventions such as outdoor spraying can still cause mortality. At the end of this immature interval, before the start of host-seeking, female mosquitoes mate. Male mosquitoes are included in the simulation to allow simulation of the mosquito population genetic structure, as well as interventions and phenomena such as release of modified males or mosquitoes with Wolbachia infection. Each female is mated once, with fertility only if the male is not sterile and there is no cytoplasmic incompatibility due to $\mathrm{Wol}$ bachia type [31]. Mating outcomes are based on the current distribution of male mosquitoes. Sterile-mated females will blood-feed, but do not produce viable eggs.

\section{Adult mosquito populations}

Host-seeking and blood-feeding are key aspects of the reproductive life of an adult female Anopheles, and these are also the key aspects for malaria transmission. After completion of post-emergence maturation, female adults enter a cycle of feeding and egg-laying which will consume the rest of their lives. Female Anopheles mosquitoes bloodfeed every $1 / \mathrm{f}=2-4$ days [11], and in the model, a fixed proportion $(=\mathrm{f} \Delta \mathrm{t})$ of all mosquitoes in a state cohort attempt to feed during a time step $\Delta t$. Subsequent versions of this model include a state with a timer for blood feed processing, which replaces the draw for fraction feeding each time step. The total number of mosquitoes in a cohort that attempt to feed during a time step are then stochastically sorted into a variety of outcomes depending on vector behaviour, host availability, and interventions, such as insecticidetreated nets (ITN) and indoor residual spraying (IRS) as described in Figure 4.

Possible outcomes of an attempted feeding include death, survival without feeding, successful feed on a human, or successful feed on an animal. A binary decision tree is created for progress through a feeding cycle as seen in Figure 4. If a feed is attempted, the first branching point is the choice of host type depending on the vector host preference, and if human is selected, the location of feeding is chosen based on the vector indoor feeding probability. Each possible choice is thus conditional on arriving at that stage of the decision tree, allowing simpler definitions of efficacy. Blocking efficacy of nets is specified as the probability that a net blocks a feed, given an attempted indoor feed on a protected human, rather than the reduction in overall successful biting. This binary structure allows logical combination of the effects of ITNs and IRS and makes it simple to add new interventions to the model. An indoor feed 


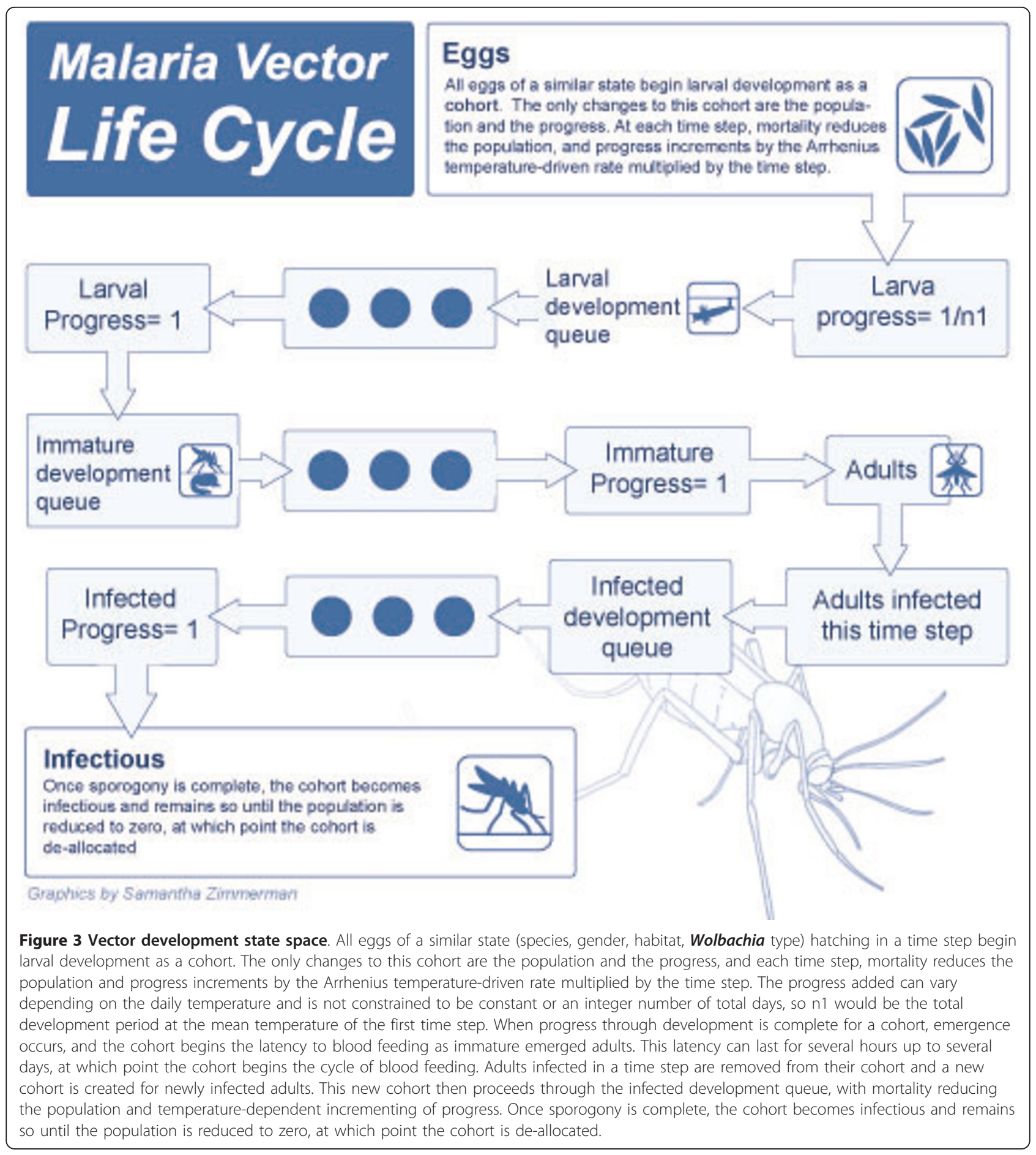

only occurs if the net does not block the feed and the treated net does not kill the mosquito. Mosquitoes that complete a feed are eligible to rest on an IRS-treated wall with a specified killing efficacy. Thus the effects of ITNs and IRS in the same house are not independent, and blocked feeds reduce the number of mosquitoes that arrive at the IRS section of the decision tree.
Successful feeds on humans have an additional draw for whether the mosquito is infected with Plasmodium or not which depends on human infectiousness, and the conditional probability of surviving a feed on an infectious individual. En route to assembling the distribution of feeding outcomes, human biting rate and entomological inoculation rate (EIR) are calculated, including all 


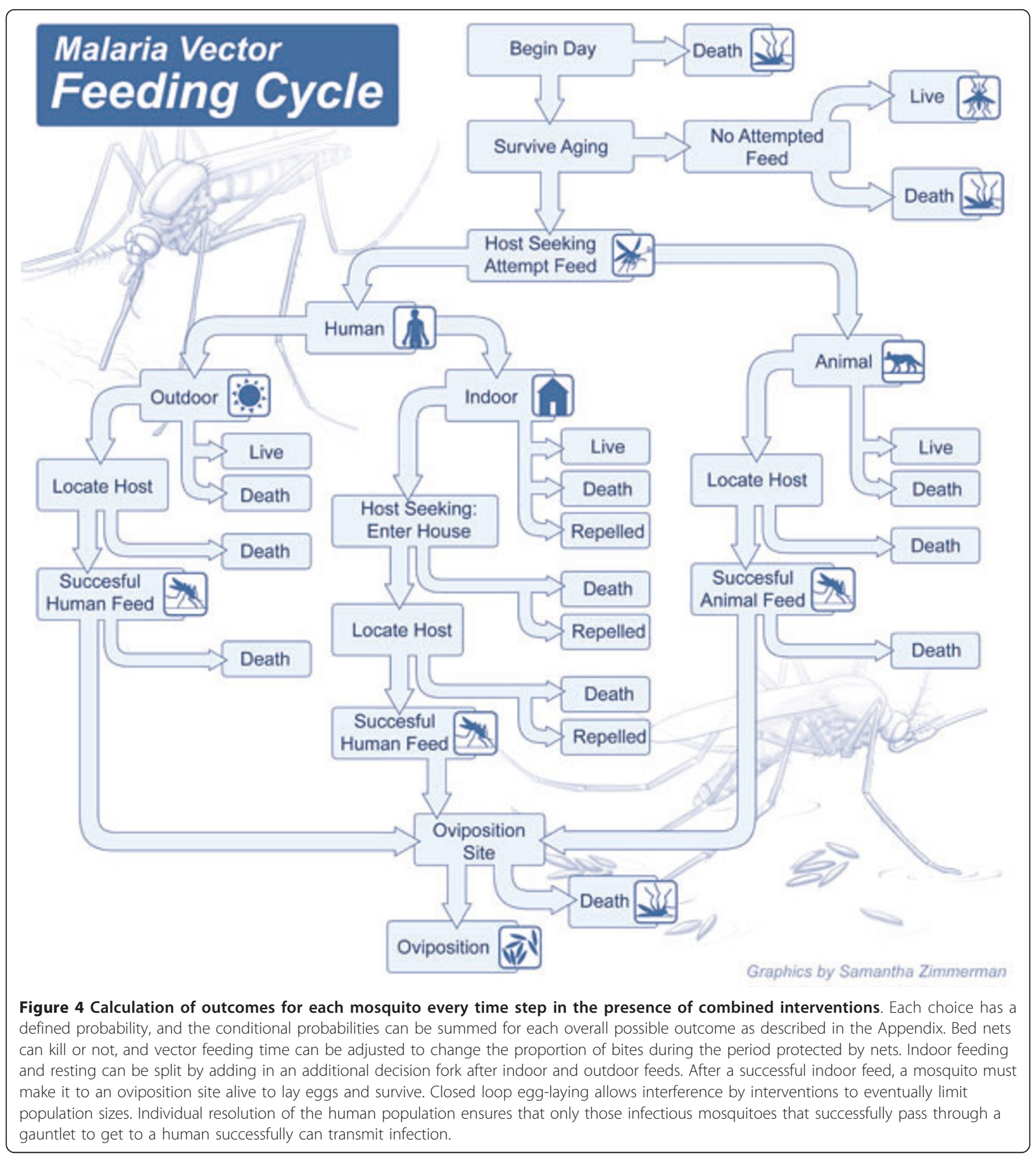

human feeds whether or not the mosquito survives, as mosquito death can occur before, during, or after feeding, but transmission can occur even for feeds which the mosquito does not survive. The presented version of the model, with a draw for number feeding each day and without the timer for blood feed processing, calculates the outcomes for the full feeding cycle including blood meal processing and oviposition survival, but assembles these into outcomes in a single calculation with eggs laid that time step for those feeds. The detailed equations for feeding outcomes are contained in Additional File 1.

The effect of a local mosquito species population on disease transmission depends on several species-specific 
characteristics. Among the most important is anthro-

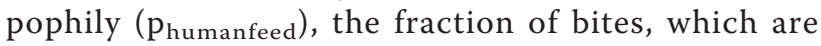
taken on a human host, or human blood index $[32,33]$. Indoor versus outdoor feeding and resting, represented as an probability of feeding indoors ( $\mathrm{p}_{\text {indoorfeeding }}$ ), is another important behaviour of the local mosquito population, especially when indoor interventions such as IRS and ITN's are introduced. Vectors which feed predominantly indoors can be decimated by these interventions, while those species which feed outdoors will not experience the same applied mortality. Indoor feeding and resting are not necessarily equal for a mosquito species, and this would be simple to implement in the present outcome calculation structures. In the presented sample simulations, indoor feeds are associated with indoor resting and outdoor feeds with outdoor resting, although a given species may have a mixed proportion of indoor and outdoor feeds.

The most important factor for baseline transmission is the adult mortality $[9,10]$, which can be calculated per day or per feeding cycle. Mosquitoes also experience additional mortality at high temperatures with low humidity [4]. The formula of Martens for daily survival to a temperature-dependent mortality rate, with $\mathrm{T}$ in Celsius, is approximated as $\left(.001 \mathrm{e}^{(\mathrm{T}-32)}\right)$, which does not have the mathematical pathologies at the roots of Martens' polynomial. Mosquitoes do exhibit age effects and senescence in laboratory settings, and senescence has been observed in field studies as well [34]. In fact, mosquitoes have not been found in the field having taken more than 14 feeds [11]. These possible effects are studied by adding age to the state space, which results in a much larger number of cohorts, and adding an agedependent mortality rate to the standard daily or feeding cycle mortality.

In the cohort model, the number of eggs laid per time step is calculated from the number of successful feeds on humans and animals occurring in that time step, with corrections for number of eggs per feeding type. There is no delay currently in the present model for egg-production, and the population growth dynamics are constrained by the days between feeds and full larval-development latencies. However, oviposition timers can be incorporated both in the individual-mosquito based simulations and in the cohort model. Determining the number of eggs from successful blood feeds allows second order effects of interventions on the mosquito population to be captured, which is not possible in models which utilize a pre-determined temporal pattern for emergence rate of mosquitoes.

\section{Infection}

A bite on an infected human can result in mosquito infection with Plasmodium, with a probability of infection dependent on a variety of human and mosquito factors [35-44]. In general, human infectivity tends to increase with gametocyte densities in a typical blood meal of 1-3 $\mu$ l [35], but this can be reduced by high parasitaemia provoking an inflammatory cytokine response $[45,46]$, by age and immunity of the human host [13], and by gametocyte-killing drug treatments [47]. Once within the mosquito gut, Plasmodium progresses through several stages of development in the mosquito finally resulting in sporozoites within the salivary glands which can infect human hosts [48]. The mosquito attempts to avoid infection through various defenses against Plasmodium gametocytes [49] and melanotic encapsulation of its oocysts [50].

The effect of weather and climate on malaria transmission is seen again in temperature-dependent latencies in sporozoite development $[4,49,51,52]$. The development times are seen in Figure 1c, and the corresponding survival probabilities are plotted in Figure 2b. These are included in traditional continuous compartmental models as a factor for mosquito survival of this latency, which is multiplied by the rate of change in infectious mosquitoes. This effect can be implemented in cyclical model as a changing probability of surviving incubation with fixed probability of surviving a feeding cycle [53]. This cohort implementation avoids instantaneous transport from susceptible to infected status, even scaled appropriately for steady state. Steady states are rare to non-existent in malaria transmission: seasonality in temperature and rainfall changes vector population sizes and infection rates, monthly rainfall for the same month varies from year to year, and human population infectiousness may not be at the same level at the same time each year, all of which may affect the impact of interventions as a function of their timing. Therefore, it is important that full latencies are enforced, with infectious mosquitoes not appearing until completion of the intervening stages.

Progress towards infectiousness is included as a state variable, and the infection state variable is not changed from infected to infectious until the progress state variable reaches completion. Enforcement of larval and immature latencies similarly captures the dynamics for population growth. Mosquitoes of the same state infected the same evening become a new state cohort in the simulation, and each time step, progress towards infectiousness is incremented by the temperature-dependent rate. At infection, the number infected is subtracted from the population of the uninfected cohort, and a new cohort is allocated with the newly infected population and zero progress towards sporogony. Upon completion, either the cohort is either merged with an identical-state infectious mosquito state cohort, or maintained separately if no identical state likely exists, as in the case of age-tracking. 
Outcome probabilities can change in response to infection status. Sporozoite infection of the salivary glands can result in increased feeding mortality when infectious $[54,55]$. Fecundity can be affected as smaller egg-batch size is observed due to maturing oocysts [56] but not salivary-gland sporozoites [57]. Once infectious, a mosquito's bites have a probability of infecting a human host, whether the mosquito survives the feed or dies during or after the feed. Probability of human infection from a sporozoite-positive bite can be calculated from field data or laboratory experiments, with a value of approximately 0.5 per bite probably being reasonable [58].

\section{Interventions}

Simulations of vector populations in the absence of interventions, such as bed nets, are important, but the purpose of the present model is to evaluate the effects of interventions singly and in combination, especially in the global eradication context. Key issues include incorporation of the effects of each of the full spectrum of interventions to examine possible effects and determining how interventions combine their effects. Some interventions target adult female Anopheles feeding, and these include insecticide treated bed nets [59-61], indoor residual spraying and screening [62]. Figure 4 shows how each intervention affects the feeding cycle as discussed above and how total outcomes can be calculated when interventions are combined, with full equations in Additional File 1.

Other interventions affect the population through the larval stage, either directly with larvicides $[63,64]$ and larval predators or indirectly through habitat destruction. Land usage modification, either intentional or unintentional, due to urbanization, agriculture, or draining swamps can have powerful effects [62,63]. Larval control options available in the model include temporary increases in larval mortality through larvicides in a subset of local habitat, repeated treatments with sufficient mortality to render a fraction of local habitat unavailable for longer intervals, or land usage reducing larval habitat long term. Depending on the option, the model either implements a temporary increase in larval mortality in a subset of the habitat carrying capacity, or proportionately reduces the habitat carrying capacity for the duration of effect.

\section{Individual mosquito model}

In addition to state cohorts, this basic model can be implemented through simulation of every individual mosquito or simulation of a subset of individual mosquitoes to represent the full population. Each mosquito's state contains the same features as the state cohort model, with status, timers for transition to adult from immature and infected to infectious, mating status and Wolbachia infection, and age. An oviposition timer to enforce a fixed feeding cycle may be included as well. If mosquitoes are sampled and a subset used to represent the local population, each sample mosquito will have an associated sampling weight as well.

\section{Setting up simulations}

Vector dynamics are simulated for single human populations well-mixed with multiple vector populations. All simulations are based upon a single-location with temperature [19] and rainfall [65] based upon lat-long (-8.5, $36.5)$ in Tanzania. Three local vector populations are simulated: An. gambiae s.s., An. arabiensis, and An. funestus. Anopheles gambiae and An. arabiensis are modelled to track the rainfall with the short temperature and humidity-dependent time constant, while An. funestus larval habitat integrates rainfall with a smaller forcing term and decays with a much slower time constant, here set to 100 days to correspond to the length of an agricultural season. The habitat scaling parameters and habitat-specific time constants were obtained by simulation of one species at a time, comparing to measurements of local EIR by species. Parameters which exhibit high uncertainty or geographic variability, such as the host preference of An. arabiensis, are studied over broad numerical ranges for their impact on results. A simplified human disease model is used in all simulations, with a constant latent period of 22 days from bite to infectiousness to mosquitoes, and exponentially-distributed period of infectiousness with mean 180 days. Infectiousness is a constant 0.2 , without development of immunity to allow resolution of vector-specific effects. Superinfection is allowed, with a maximum of five simultaneous infections. General model and simulationspecific parameters and their values are summarized in Table 1.

\section{Results and Discussion}

Baseline vector population and transmission dynamics are simulated for the single location simulation described in Methods. The habitat scaling parameters are varied to show different baseline EIRs with the same weather-driven seasonality. The total vector population when summed across all three species is seen in Figure $5 \mathrm{a}$, with the rainfall patterns and temperature in $5 \mathrm{~b}$. Figure 5a shows the effect of scaling the time series of available larval habitat, Figure $5 \mathrm{c}$ presents the resulting sporozoite rates, and Figure $5 \mathrm{~d}$ the entomological inoculation rate, which represents the infectious bites received per person per night. If mortality increases as a function of age, the total population numbers do not change greatly, but the sporozoite rate drops due to the suppression of the older part of the mosquito age- 
Table 1 Model and simulation parameters

\begin{tabular}{|c|c|c|}
\hline Parameter & Value used in simulations & Source, notes \\
\hline Habitat scalars K $K_{\text {temp }}$ & $1.25 \times 10^{9}$ for gambiae ss and $11.25 \times 10^{9}$ for arabiensis & Fit to site-specific data through simulation \\
\hline Habitat scalar $\mathrm{K}_{\text {semi }}$ & $6 \times 10^{8}$ for funestus & Fit to site-specific data through simulation \\
\hline $\begin{array}{l}\text { Habitat time constants } \\
\text { ktempdecay and } \tau_{\text {semi }}\end{array}$ & $\begin{array}{l}0.05\left(k_{\text {tempdecay }}\right) \\
0.01 / \text { day }\left(\tau_{\text {semi }}\right)\end{array}$ & Fit through simulation \\
\hline $\begin{array}{l}\text { Larval development Arrhenius } \\
\text { parameters } a_{1}, a_{2}\end{array}$ & $8.42 \times 10^{10}, 8.3 \times 10^{3}$ & Fit to traditional curve in [4] \\
\hline $\begin{array}{l}\text { Incubation period Arrhenius } \\
\text { parameters } a_{1}, a_{2}\end{array}$ & $1.17 \times 10^{11}, 8.4 \times 10^{3}$ & Fit to traditional curve in [4] \\
\hline Duration of immature & 4 days & $\begin{array}{l}\text { Not a very sensitive parameter, given the habitat fit to } \\
\text { adult population }\end{array}$ \\
\hline Days between feeds & 3 days & 2-4 days [11] \\
\hline Human blood index & 0.95 for gambiae ss and funestus, variable for arabiensis & $\begin{array}{l}\text { [69]. The uncertain value for arabiensis is the focus of } \\
\text { detailed analyses. }\end{array}$ \\
\hline Indoor feeding & $\begin{array}{l}\text { To explore effects, gambiae and funestus were set as highly } \\
\text { endophilic and arabiensis was varied }\end{array}$ & \\
\hline $\begin{array}{l}\text { Female eggs per female } \\
\text { oviposition }\end{array}$ & 100 & A more accurate value would be closer to 80 \\
\hline $\begin{array}{l}\text { Modification of egg batch } \\
\text { size for infection }\end{array}$ & 0.8 & \\
\hline Adult life expectancy & 10 days & [9-11] \\
\hline Transmission modifier b & 0.5 & [58] \\
\hline Mosquito infection modifier c & 0.2 & $\begin{array}{l}\text { Will in reality depend on human infectiousness [36], } \\
\text { here set to be uniform for simplicity }\end{array}$ \\
\hline Human feeding mortality & 0.1 & Uncertain \\
\hline $\begin{array}{l}\text { Human feeding mortality for } \\
\text { sporozoite positive }\end{array}$ & 0.15 & Uncertain \\
\hline
\end{tabular}

distribution. Note that increasing larval habitat has a second order effect on EIR by allowing the human prevalence to rise earlier in the season, which increases the infectivity of the human population to vectors.

Further simulations demonstrate effects of combined vector control interventions such as insecticide-treated nets (ITN), indoor residual spraying (IRS), larval control, and space spraying. Figures 6 a, c, e show the changes in vector dynamics as coverage with perfect IRS is increased, killing all indoor feeding mosquitoes and maintained at full efficacy for the specified coverage without decay. In addition, all mosquitoes are set to feed indoors, making this an unrealistic scenario, but a useful boundary case showing the maximum possible effect. Larval habitat is set to 3.0 for all three species, and the simple human disease model is used. The detailed model outputs can be used to determine the change in entomologic inoculation rate as described in [9]. These results can be compared to field results for bed net campaigns in the presence of multiple vector species [66]. The size of the local vector population is reduced, but the effects on sporozoite rate and EIR are much more dramatic for several reasons, especially the restructuring of the mosquito population age distribution. The higher mortality results in fewer old mosquitoes in the population, which is the segment of the population with sporozoites. The older cohorts are thus responsible for the major portion of EIR but only minor portions of adult populations, human biting, and fecundity. In many cases, larval habitat remains the limiting factor in determining the number of emerging mosquitoes and interventions primarily act through adult mortality, but at high IRS or ITN coverage levels, it is possible in simulations to reduce emergence rate by limiting successful feeds. This phenomenon has been seen in high-coverage field studies [67], and the disappearance of An. funestus from parts of its earlier habitat as intervention coverage increases is the extreme limit of this phenomenon. The simulations are repeated for IRS with 0.6 killing of post-feeding mosquitoes and results are shown in Figures $6 \mathrm{~b}, \mathrm{~d}$, f. The effects on adult vectors and sporozoite rate are reduced, and these reductions are compounded in the effect on EIR. Feeds in houses with IRS now have a 40 percent survival probability in contrast to the 0 percent survival in the previous simulations. Thus for 60 percent coverage, the survival for indoor feeds on the population is now 64 percent instead of 40 percent, and the probability of surviving three feeds rises to 26 percent from 6 percent. 

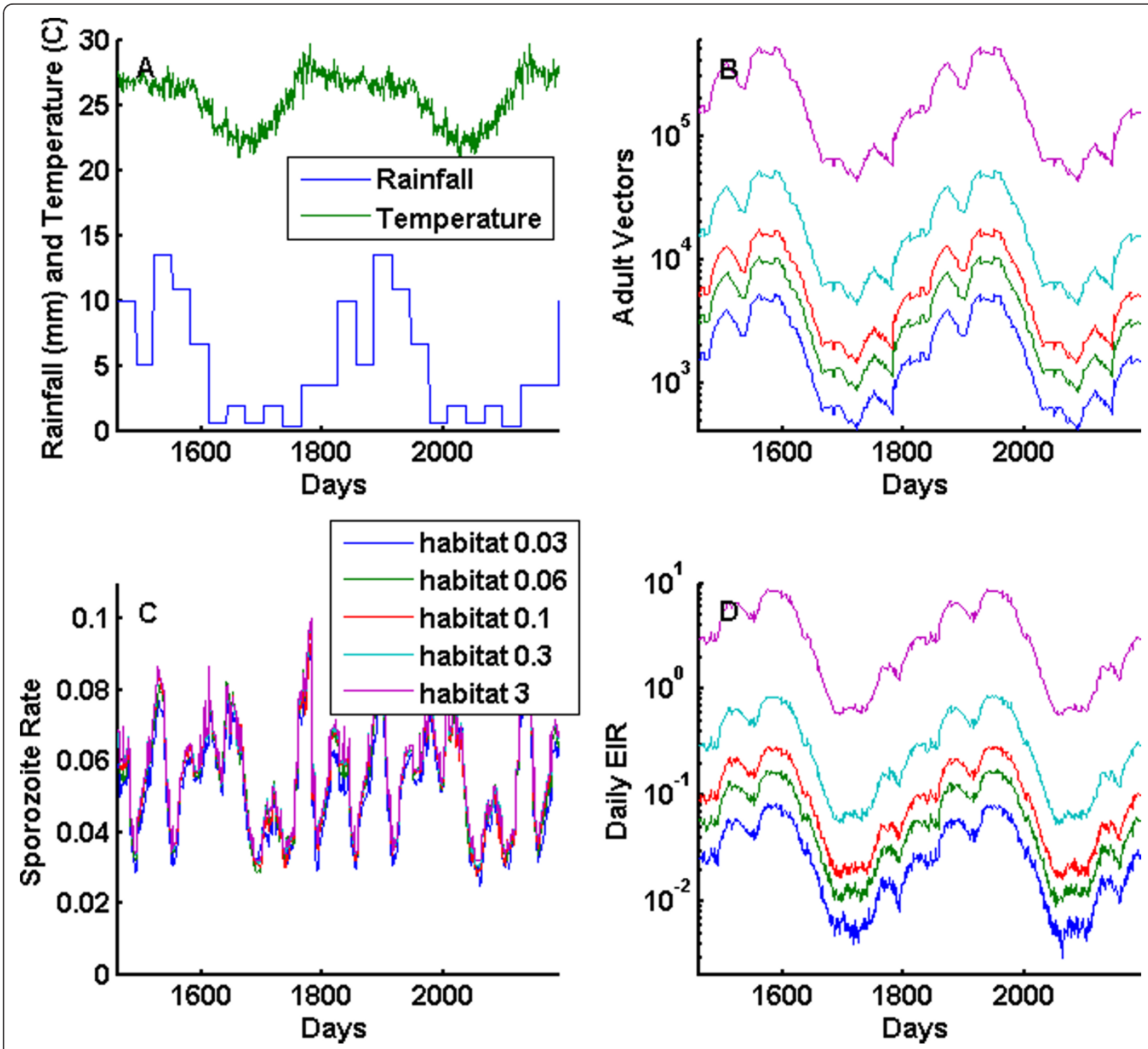

Figure 5 Baseline population dynamics summed over local populations of An. gambiae ss, An. funestus, and An. arabiensis for different larval habitat multipliers. a) Local weather will drive both the temporary and semi-permanent larval habitats. b) The adult vector population changes as a function of the scaling of the larval habitat carrying capacity, which is driven by local weather. c) The sporozoite rate of mosquito population changes in response to the changing age structure of the vector population over the course of two years. d) Daily ElR combines the adult vector population and the sporozoite rate.

The effects of co-varying IRS and ITN coverage are studied and many simulations are run over sections of campaign and parameter space, with each simulation a trial for local elimination success or failure. These trials can be used to estimate the probability of local elimination as a function over campaign or parameter space. In Figure 7, coverage with IRS and ITN is varied, and the trials are assembled into plots, which map the regions of high probability of success and low probability of success. For purposes of this demonstration, both IRS and ITN kill every single relevant mosquito $\left(\mathrm{p}_{\mathrm{kill}}\right.$, ITN $=1$ and $\mathrm{p}_{\text {kill, }}$ IRSpostfeed $=1$ ) and do not decay. All three mosquito species are simulated to feed indoors and take 95 percent of their blood meals on human hosts. The larval habitat scaling is set to 1.0 , and the simple human disease model is used. Given these assumptions, it is not surprising that the region of success is large. Decreasing pkill, ITN and $p_{\text {kill, IRSpostfeed }}$ to 0.6 and maintaining bed nets at $100 \%$ blocking of indoor feeding produces the 2D plot in Figure 7c. High coverage of bed nets still successfully locally eliminates the disease, since all feeds in this simulation occur indoors at night, but this is a 


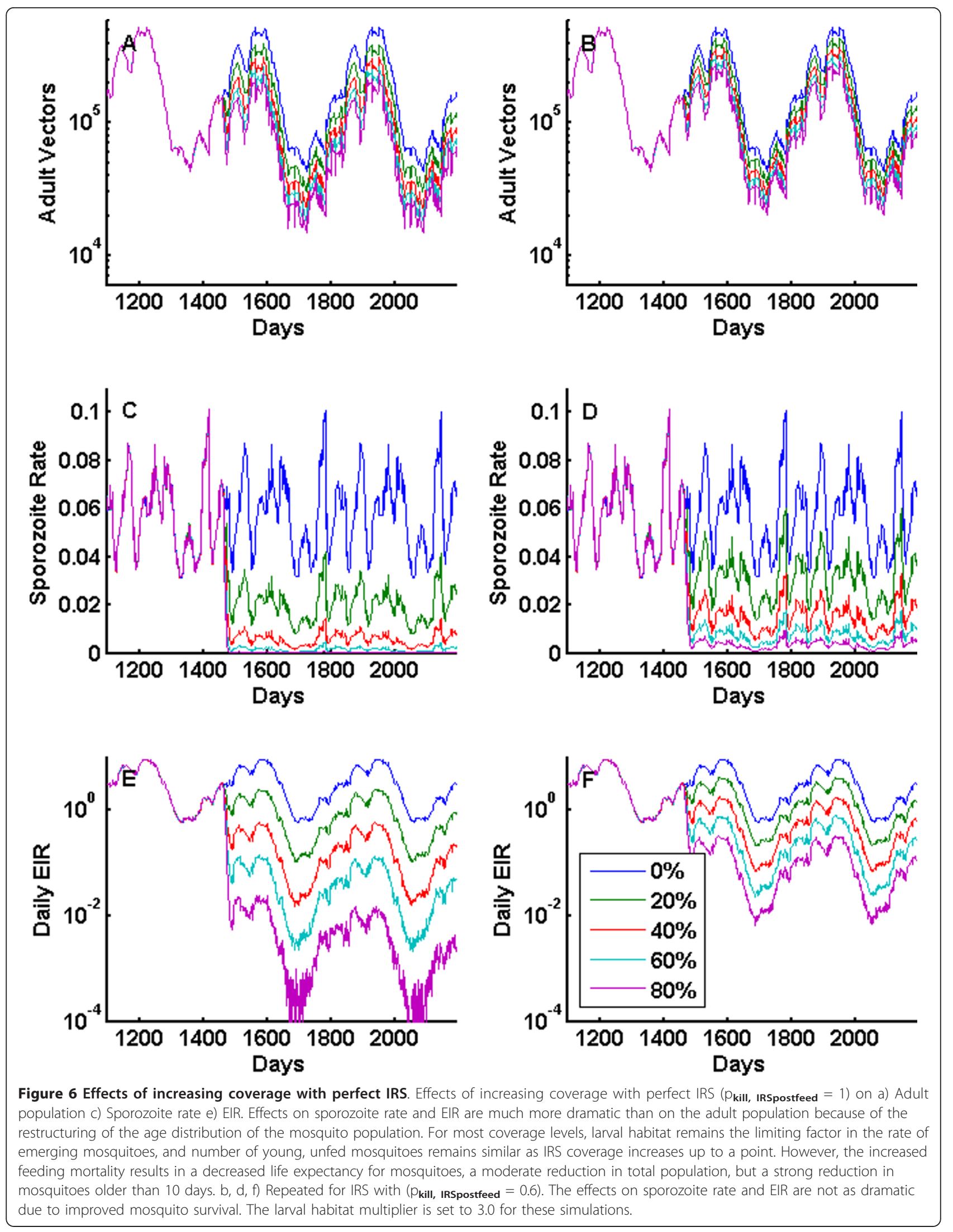




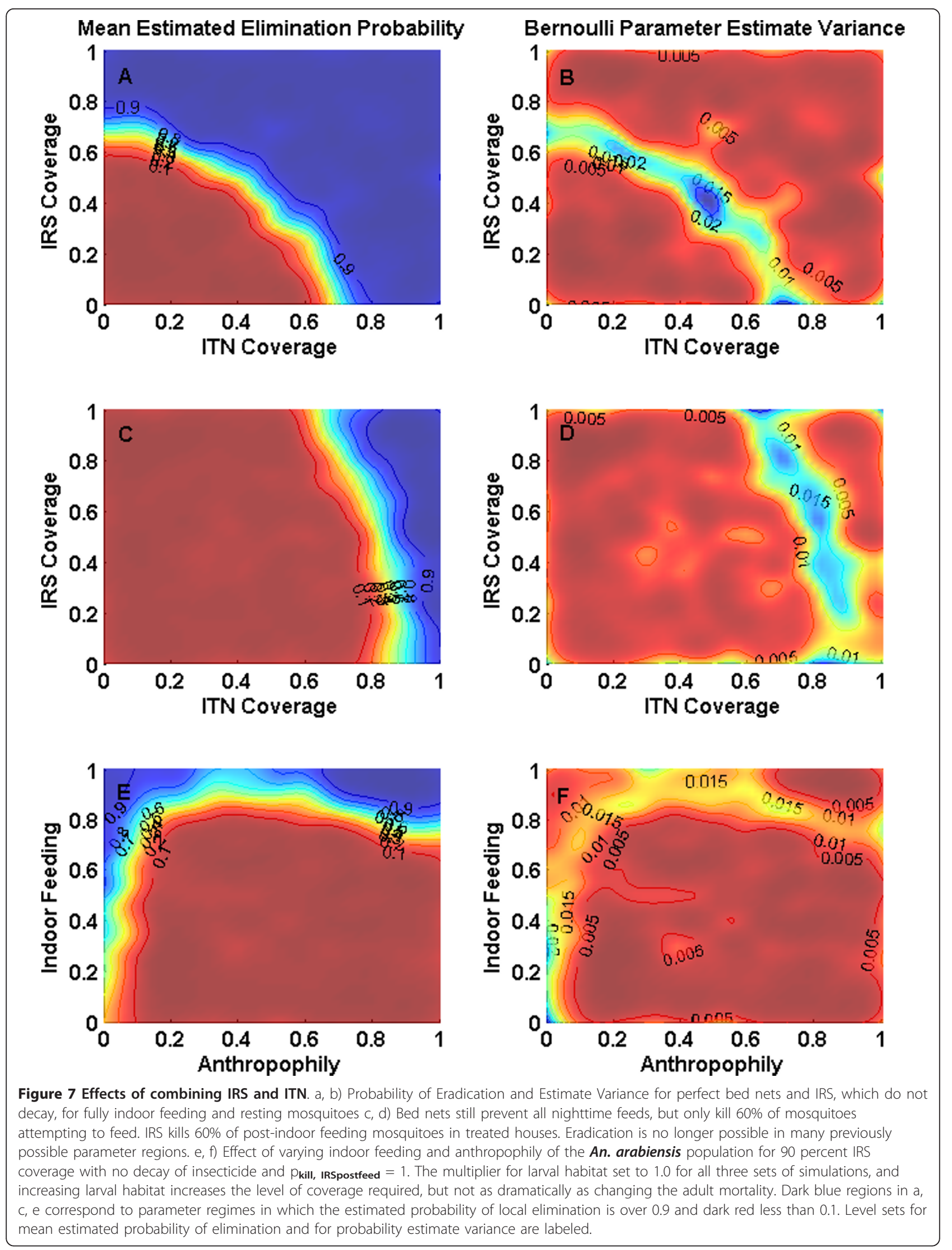


quadratic effect of blocking part of the population from acquisition and transmission, while the exponential effect of increasing local mosquito mortality is drastically reduced with dramatic effects. Even with full IRS coverage and all mosquitoes feeding indoors, a reduced killing efficacy may not permit local elimination without supplemental interventions. The reduced efficacy is intended to represent an effect of insecticide resistance, and the effect on elimination has important implications for campaigns.

Changes in vector behaviours such as indoor feeding and anthropophily change the results drastically with several key implications for eradication. The previous system is rerun with IRS at 90 percent coverage with full mosquito post-feeding mortality for indoor feeds in treatedhouses, and the human feeding and indoor feeding preferences of the local arabiensis population are varied. Changes in indoor feeding have a dramatic effect on campaign success as seen in Figure 7 e, f, as would be expected for interventions which only affect indoor feeding mosquitoes. Decreasing anthropophily is often viewed as decreasing transmission, but in the presence of high intervention coverage, decreasing anthropophily reduces the mortality during sporogony, allowing a higher proportion of infected mosquitoes to complete sporogony and thereby reducing the probability of campaign success. Feeds on animals during sporogony are safe compared to the protected human feeds and increase the probability of surviving the multiple feeds during sporogony and becoming infectious. At lower anthropophily, fewer mosquitoes become infected and infectious mosquitoes bite humans less frequently, and this effect eventually wins and probability of success increases with decreasing anthropophily below a certain value. Detailed model representations of vector behaviour help explain the failure of elimination campaigns which only targeted indoor feeding. For eradication to succeed, the full transmission cycle must be sufficiently broken, which may involve targeting outdoor-feeding mosquitoes in some areas.

Other available but currently less-used options for vector control include larvicides and space spraying for targeting larval and adult populations, respectively. Figure 8 shows results for simulations of larval control on the left and adult-targeting space spraying on the right. In the presented simulations, larval control is simulated as a temporary decrease in the larval habitat carrying capacity for a specified duration, such as a 30 percent reduction for 180 days. This produces decreases in the adult population, but not in the adult age-structure-driven sporozoite rate, except for transients at the start and conclusion of larval control. Thus the effects on EIR tend to be linear, but this should not be ignored as larval control may be one of the only ways to target outdoor feeding mosquitoes.
In order to achieve local elimination of malaria in areas with high rates of outdoor feeding by the local vector populations, some form of control of outdoor mosquitoes may be necessary. Available options can be costly and logistically difficult, and studies of their effects can place constraints on required target efficacy, duration, and frequency of such efforts. In the model, space spraying increases the mortality for all adult mosquitoes, regardless of the stage in the feeding cycle. The artificial daily mortality probability is calculated as 1-exp (-killrate* $\Delta \mathrm{t}$ ), so that a killrate of 1.0 will tend to kill approximately 63 percent of adult vectors. As seen in Figure 8 , even one day of spraying with a high killrate can produce a large several week drop in EIR as it takes time for the newly emerging mosquitoes to become infected and infectious. Longer duration efforts, such as repeated spraying with a given knockdown of adult vectors each cycle, increase the daily mortality and reduce the number of adult vectors, reshape the age structure and reduce the sporozoite rate and achieve a strong multiplicative decrease on the EIR. Such maintained repetitive space spraying with non-residual insecticides may be logistically difficult, but it could provide leverage on the outdoor feeding population if other options fall through. Simulations such as these can estimate the effects on vector populations for a given input efficacy, duration, and frequency of application, providing inputs to cost-effectiveness analyses.

\section{Conclusions}

The present model creates a flexible framework for exploring the effects of combined vector control interventions on vector population dynamics and disease transmission. Campaigns with IRS and ITN are studied and success of elimination is seen to depend on coverage and efficacy as expected, but also on mosquito behaviour. As $A n$. arabiensis outdoor feeding increases, interventions which target indoor feeding become less effective. Decreasing anthropophily from unity at high coverage decreases the rate of killing mosquitoes during sporogony, initially reducing elimination success, but this returns to the expected relationship of less anthropophily increasing elimination success as anthropophily continues to decline to low levels. Climate and weather data with high spatial resolution can help predict spatial and temporal patterns of vector dynamics and assist the rational planning of regional campaigns, especially when included combined with a population transmission model $[16,17,19]$.

Further work will exhibit the effect of vector migration and seasonality on interventions such as locally-applied larvicides. Understanding the role and scale of migration is important for estimating effect of adult vector interventions [61,67] and larval control interventions 


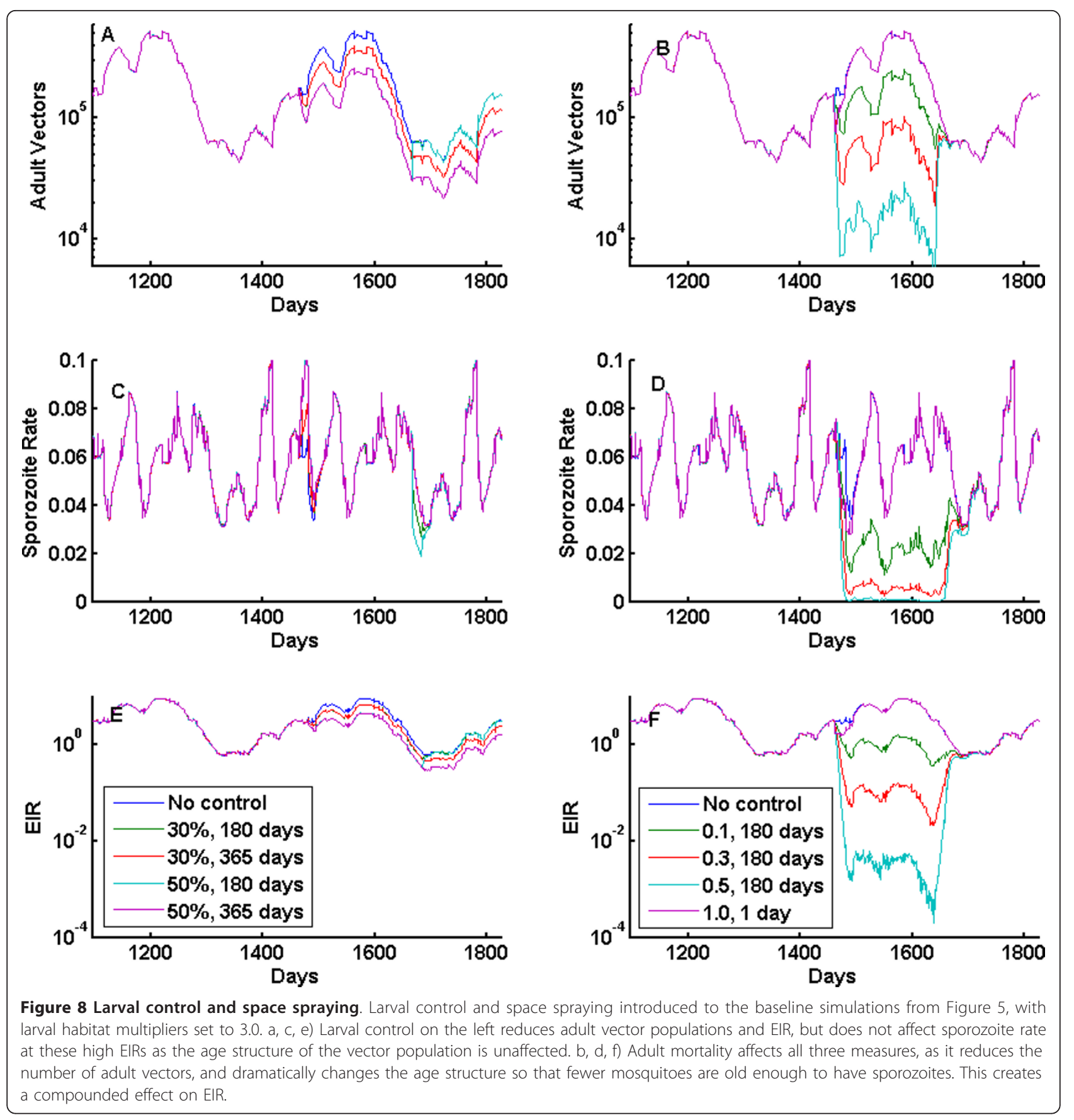

$[9,59,63,64]$. The model supports spatially-distributed simulations, and future work will explore the effects of human and vector migration on spatial transmission. Each individual in the simulation has a relative biting rate, which allows study of heterogeneous biting as has been done [37], and link to studies of attractiveness of humans to mosquitoes versus level of malaria infection [68]. Improvements will be made to the density-dependence effects of larval dynamics and models for habitat calculations will be improved. Other future work will model the response of vector populations to applied pressure, such as the lower anthropophily of $A n$. gambiae ss in The Gambia after extended bed net pressure [32]. Changes in behavior or development of insecticide resistance require careful consideration to ensure success of a designed Eradication campaign. The effects of such changes can be seen in the above-presented results, and the present model has the flexibility to incorporate dynamic changes. The modular structure of the model and the implementation of the life and feeding cycles 
also make it simple to add new potential interventions to the model. Other species are easy to add provided that an applicable habitat model has been developed. Future work can couple this detailed vector transmission model to a more detailed model of human disease and immunity.

Campaigns must address the ecology and behaviour of local mosquito populations in order to ensure that sufficient resources with broad enough effects for all relevant components of the local mosquito populations are introduced. A one-size-fits-all campaign is not optimal, being wasteful in some circumstances and insufficient in others; local tailoring and design are important. Modelling can be used to estimate the risk of disease transmission given reintroduction to areas that had achieved local elimination before their neighbours [6]. Modelling at this level of detail also serves to identify basic data gaps such as local vector ecology and behaviour that must be answered to reduce uncertainty of campaign success. Numerical studies can reveal to which parameters the results of interest are most sensitive, and such parameters which are also poorly constrained by data or are highly geographically-variable can then be highlighted as important data gaps. Modelling studies can also explore the extent to which more through and extensive campaigns can overwhelm data uncertainties and achieve more robust success.

\section{Additional material}

Additional file 1: Detailed equations for A malaria transmissiondirected model of mosquito life cycle and ecology. The detailed calculations of vector feeding outcomes for feeds on a single individual and on the full local human population are provided.

\begin{abstract}
Acknowledgements
The author thanks Bill and Melinda Gates for their active support of this work and their sponsorship through the Global Good Fund. This work was performed at Intellectual Ventures Laboratories, and useful discussions with colleagues in this Program, the Malaria Program of the Bill and Melinda Gates Foundation, MalERA, and the Vector Control Development Network are likewise greatly appreciated.
\end{abstract}

\section{Competing interests}

The authors declare that they have no competing interests.

Received: 19 July 2011 Accepted: 17 October 2011

Published: 17 October 2011

\section{References}

1. Snow RW, Guerra CA, Noor AM, Myint HY, Hay SI: The global distribution of clinical episodes of Plasmodium falciparum malaria. Nature 2005, 434:214-217.

2. Jacob BG, Muturi E, Halbig P, Mwangangi J, Wanjogu RK, Mpanga E, Funes J, Shililu J, Githure J, Regens JL, Novak RJ: Environmental abundance of Anopheles (Diptera: Culicidae) larval habitats on land cover change sites in Karima Village, Mwea Rice Scheme, Kenya. Am J Trop Med Hyg 2007, 76:73-80.
3. De Castro MC, Yamagata Y, Mtasiwa D, Tanner M, Utzinger J, Keiser J, Singer BH: Integrated urban malaria control: a case study in Dar Es Salaam, Tanzania. Am J Trop Med Hyg 2004, 71:103-117.

4. Craig MH, Snow RW, le Sueur D: A climate-based distribution model of malaria transmission in sub-Saharan Africa. Parasitol Today 1999, 15:105-111.

5. Hay SI, Guerra CA, Gething PW, Patil AP, Tatem AJ, Noor AM, Kabaria CW, Manh BH, Elyazar IR, Brooker S, Smith DL, Moyeed RA, Snow RW: A world malaria map: Plasmodium falciparum endemicity in 2007. PLoS Med 2009, 6:e1000048.

6. Cohen JM, Smith DL, Vallely A, Taleo G, Malefoasi G, Sabot O: Holding the Line. In Shrinking the Malaria Map: A Prospectus on Malaria Elimination. Edited by: Feachem RGA, Phillips AA, Targett GA, Group ME. San Francisco: The Global Health Group: UCSF Global Health Sciences; 2009:40-60.

7. Ross R: The prevention of malaria New York; : E.P. Dutton \& company; 1910.

8. Macdonald G: The epidemiology and control of malaria London, New York: Oxford University Press; 1957.

9. Smith DL, McKenzie FE: Statics and dynamics of malaria infection in Anopheles mosquitoes. Malar J 2004, 3:13.

10. Saul AJ, Graves PM, Kay BH: A cyclical feeding model for pathogen transmission and its application to determine vectorial capacity from vector infection rates. J Applied Ecol 1990, 27:123-133.

11. Killeen G, McKenzie F, Foy B, Schieffelin C, Billingsley P, Beier J: A simplified model for predicting malaria entomologic inoculation rates based on entomologic and parasitologic parameters relevant to control. Am J Trop Med Hyg 2000, 62:535-544.

12. Yazoume $Y$, Hoshen M, Kyobutungi $C$, Louis VR, Sauerborn R: Local scale prediction of Plasmodium falciparum malaria transmission in an endemic region using temperature and rainfall. Global Health Action 2009, 2.

13. Killeen GF, Ross A, Smith T: Infectiousness of malaria-endemic human populations to vectors. Am J Trop Med Hyg 2006, 75:38-45.

14. Bomblies A, Duchemin J-B, Eltahir EAB: Hydrology of malaria: Model development and application to a Sahelian village. Water Resour Res 2008, 44:W12445.

15. Depinay J-M, Mbogo C, Killeen G, Knols B, Beier J, Carlson J, Dushoff J, Billingsley P, Mwambi H, Githure J, Toure AM, McKenzie FE: A simulation model of African Anopheles ecology and population dynamics for the analysis of malaria transmission. Malar J 2004, 3:29.

16. Filipe JA, Riley EM, Drakeley CJ, Sutherland CJ, Ghani AC: Determination of the processes driving the acquisition of immunity to malaria using a mathematical transmission model. PLoS Comput Biol 2007, 3:e255.

17. Griffin JT, Hollingsworth TD, Okell LC, Churcher TS, White M, Hinsley W, Bousema T, Drakeley CJ, Ferguson NM, Basáñez M-G, Ghani AC: Reducing Plasmodium falciparum malaria transmission in Africa: a model-based evaluation of intervention strategies. PLoS Med 2010, 7:e1000324.

18. Smith T, Maire N, Ross A, Penny M, Chitnis N, Schapira A, Studer A, Genton B, Lengeler C, Tediosi F, de Savigny D, Tanner M: Towards a comprehensive simulation model of malaria epidemiology and control. Parasitology 2008, 135:1507-1516.

19. Smith T, Killeen GF, Maire N, Ross A, Molineaux L, Tediosi F, Hutton G, Utzinger J, Dietz K, Tanner M: Mathematical modeling of the impact of malaria vaccines on the clinical epidemiology and natural history of Plasmodium falciparum malaria: Overview. Am J Trop Med Hyg 2006, 75:1-10.

20. Magori K, Legros M, Puente ME, Focks DA, Scott TW, Lloyd AL, Gould F: Skeeter Buster: a stochastic, spatially explicit modeling tool for studying Aedes aegypti population replacement and population suppression strategies. PLoS Negl Trop Dis 2009, 3:e508.

21. Simard F, Ayala D, Kamdem GC, Pombi M, Etouna J, Ose K, Fotsing JM, Fontenille D, Besansky NJ, Costantini C: Ecological niche partitioning between Anopheles gambiae molecular forms in Cameroon: the ecological side of speciation. BMC ECOl 2009, 9:17.

22. Fillinger $U$, Sonye $G$, Killeen GF, Knols BGJ, Becker N: The practical importance of permanent and semipermanent habitats for controlling aquatic stages of Anopheles gambiae sensu lato mosquitoes: operational observations from a rural town in western Kenya. Trop Med Int Health 2004, 9:1274-1289.

23. Koenraadt CJ, Githeko AK, Takken W: The effects of rainfall and evapotranspiration on the temporal dynamics of Anopheles gambiae s.s. and Anopheles arabiensis in a Kenyan village. Acta Trop 2004, 90:141-153. 
24. Bomblies A, Duchemin J-B, Eltahir E: A mechanistic approach for accurate simulation of village scale malaria transmission. Malar J 2009, 8:223.

25. Shaman J, Spiegelman M, Cane M, Stieglitz M: A hydrologically driven model of swamp water mosquito population dynamics. Ecological Modelling 2006, 194:395-404.

26. Guelbeogo W, Sagnon NF, Grushko O, Yameogo M, Boccolini D, Besansky N, Costantini C: Seasonal distribution of Anopheles funestus chromosomal forms from Burkina Faso. Malar J 2009, 8:239.

27. Gillies MT, De Meillon B: The Anophelinae of Africa, South of the Sahara Johannesburg, South Africa: South African Institute for Medical Research; 1968.

28. Paaijmans KP, Wandago MO, Githeko AK, Takken W: Unexpected high losses of Anopheles gambiae larvae due to rainfall. PLOS One 2007, 2: e1146.

29. Mutuku FM, Bayoh MN, Gimnig JE, Vulule JM, Kamau L, Walker ED, Kabiru E, Hawley WA: Pupal habitat productivity of Anopheles gambiae complex mosquitoes in a rural village in western Kenya. Am J Trop Med Hyg 2006, 74:54-61.

30. Clements AN: The Biology of Mosquitoes, Volume 1: Development, Nutrition, and Reproduction New York: CABI Publishing; 2000

31. Dobson SL, Fox CW, Jiggins FM: The effect of Wolbachia-induced cytoplasmic incompatibility on host population size in natural and manipulated systems. Proc R Soc London Series B: Biol Sciences 2002, 269:437-445

32. Killeen GF, McKenzie FE, Foy BD, Bogh C, Beier JC: The availability of potential hosts as a determinant of feeding behaviours and malaria transmission by African mosquito populations. Trans $R$ Soc Trop Med Hyg 2001, 95:469-476.

33. Saul A: Zooprophylaxis or zoopotentiation: the outcome of introducing animals on vector transmission is highly dependent on the mosquito mortality while searching. Malar J 2003, 2:32.

34. Clements AN, Paterson GD: The analysis of mortality and survival rates in wild populations of mosquitoes. J Applied Ecol 1981, 18:373-399.

35. Jeffery GM, Eyles DE: Infectivity to mosquitoes of Plasmodium falciparum as related to gametocyte density and duration of infection. Am J Trop Med Hyg 1955, 4:781-789.

36. Sinden RE, Butcher GA, Billker O, Fleck SL: Regulation of infectivity of Plasmodium to the mosquito vector. Adv Parasitol 1996, 38.53-117.

37. Smith DL, McKenzie FE, Snow RW, Hay Sl: Revisiting the basic reproductive number for malaria and its implications for malaria control. PLOS Biol 2007, 5:e42.

38. Githeko AK, Brandling-Bennett AD, Beier M, Atieli F, Owaga M, Collins FH: The reservoir of Plasmodium falciparum malaria in a holoendemic area of western Kenya. Trans R Soc Trop Med Hyg 1992, 86:355-358.

39. Bonnet S, Gouagna C, Safeukui I, Meunier J-Y, Boudin C: Comparison of artificial membrane feeding with direct skin feeding to estimate infectiousness of Plasmodium falciparum gametocyte carriers to mosquitoes. Trans R Soc Trop Med Hyg 2000, 94:103-106.

40. Smalley ME, Sinden RE: Plasmodium falciparum gametocytes: their longevity and infectivity. Parasitology 1977, 74:1-8

41. Schneider P, Bousema JT, Gouagna LC, Otieno S, van de Vegte-Bolmer M, Omar SA, Sauerwein RW: Submicroscopic Plasmodium falciparum gametocyte densities frequently result in mosquito infection. Am J Trop Med Hyg 2007, 76:470-474.

42. Muirhead-Thomson RC: The malarial infectivity of an African village population to mosquitoes (Anopheles gambiae); a random xenodiagnostic survey. Am J Trop Med Hyg 1957, 6:971-979.

43. Drakeley C, Sutherland C, Bousema JT, Sauerwein RW, Targett GAT: The epidemiology of Plasmodium falciparum gametocytes: weapons of mass dispersion. Trends Parasitol 2006, 22:424-430.

44. Bonnet S, Gouagna LC, Paul RE, Safeukui I, Meunier JY, Boudin C: Estimation of malaria transmission from humans to mosquitoes in two neighbouring villages in south Cameroon: evaluation and comparison of several indices. Trans R Soc Trop Med Hyg 2003, 97:53-59.

45. Naotunne TS, Karunaweera ND, Del Giudice G, Kularatne MU, Grau GE Carter R, Mendis KN: Cytokines kill malaria parasites during infection crisis: extracellular complementary factors are essential. J Exp Med 1991, 173:523-529.

46. Naotunne TS, Karunaweera ND, Mendis KN, Carter R: Cytokine-mediated inactivation of malarial gametocytes is dependent on the presence of white blood cells and involves reactive nitrogen intermediates. Immunology 1993, 78:555-562.

47. Okell L, Drakeley C, Ghani A, Bousema T, Sutherland C: Reduction of transmission from malaria patients by artemisinin combination therapies: a pooled analysis of six randomized trials. Malar J 2008, 7:125

48. Al-Olayan EM, Beetsma AL, Butcher GA, Sinden RE, Hurd H: Complete Development of mosquito phases of the malaria parasite in vitro. Science 2002, 295:677-679.

49. Beier JC: Malaria parasite development in mosquitoes. Ann Rev Entomol 1998, 43:519-543.

50. Gorman MJ, Cornel AJ, Collins FH, Paskewitz SM: A shared genetic mechanism for melanotic encapsulation of CM-Sephadex beads and a malaria parasite, Plasmodium cynomolgi B, in the mosquito, Anopheles gambiae. Exp Parasitol 1996, 84:380-386.

51. Macdonald G: Theory of the eradication of malaria. Bull World Health Organ 1956, 15:369-387.

52. Afrane YA, Little TJ, Lawson BW, Githeko AK, Yan G: Deforestation and vectorial capacity of Anopheles gambiae Giles mosquitoes in malaria transmission, Kenya. Emerg Infect Dis 2008, 14:1533-1538.

53. Burkot TR, Graves PM, Paru R, Battistutta D, Barnes A, Saul A: Variations in malaria transmission rates are not related to anopheline survivorship per feeding cycle. Am J Trop Med Hyg 1990, 43:321-327.

54. Wekesa JW, Copeland RS, Mwangi RW: Effect of Plasmodium falciparum on blood feeding behavior of naturally infected anopheles mosquitoes in western Kenya. Am J Trop Med Hyg 1992, 47:484-488.

55. Anderson RA, Knols BGJ, Kolella JC: Plasmodium falciparum sporozoites increase feeding-associated mortality of their mosquito hosts Anopheles gambiae s.l. Parasitology 2000, 120:329-333.

56. Carwardine SL, Hurd H: Effects of Plasmodium yoelii nigeriensis infection on Anopheles stephensi egg development and resorption. Med Vet Entomol 1997, 11:265-269.

57. Hogg JC, Hurd $\mathrm{H}$ : The effects of natural Plasmodium falciparum infection on the fecundity and mortality of Anopheles gambiae s. I. in north east Tanzania. Parasitology 1997, 114:325-331.

58. Smith DL, Dushoff J, Snow RW, Hay SI: The entomological inoculation rate and Plasmodium falciparum infection in African children. Nature 2005, 438:492-495

59. Fillinger U, Ndenga B, Githeko A, Lindsay SW: Integrated malaria vector control with microbial larvicides and insecticide-treated nets in western Kenya: a controlled trial. Bull World Health Organ 2009, 87:655-665, vVv.

60. Killeen GF, Smith TA, Ferguson HM, Mshinda H, Abdulla S, Lengeler C, Kachur SP: Preventing childhood malaria in Africa by protecting adults from mosquitoes with insecticide-treated nets. PLoS Med 2007, 4:e229.

61. Gimnig JE, Vulule JM, Lo TQ, Kamau L, Kolczak MS, Phillips-Howard PA Mathenge EM, ter Kuile FO, Nahlen BL, Hightower AW, Hawley WA: Impact of permethrin-treated bed nets on entomologic indices in an area of intense year-round malaria transmission. Am J Trop Med Hyg 2003, 68:16-22.

62. Snowden F: The conquest of malaria: Italy, 1900-1962 New Haven (CT): Yale University Press; 2006.

63. Fillinger U, Kannady K, William G, Vanek M, Dongus S, Nyika D, Geissbuhler Y, Chaki P, Govella N, Mathenge E, et al: A tool box for operational mosquito larval control: preliminary results and early lessons from the Urban Malaria Control Programme in Dar es Salaam, Tanzania. Malar J 2008, 7:20.

64. Killeen $G$, Fillinger $U$, Knols B: Advantages of larval control for African malaria vectors: Low mobility and behavioural responsiveness of immature mosquito stages allow high effective coverage. Malar J 2002, $1: 8$

65. Full Data Reanalysis. [http://gpcc.dwd.de]

66. Killeen G, McKenzie F, Foy B, Schieffelin C, Billingsley P, Beier J: The potential impact of integrated malaria transmission control on entomologic inoculation rate in highly endemic areas. Am J Trop Med Hyg 2000, 62:545-551.

67. Gimnig JE, Kolczak MS, Hightower AW, Vulule JM, Schoute E, Kamau L, Phillips-Howard PA, Ter Kuile FO, Nahlen BL, Hawley WA: Effect of permethrin-treated bed nets on the spatial distribution of malaria vectors in western Kenya. Am J Trop Med Hyg 2003, 68:115-120.

68. Lacroix R, Mukabana WR, Gouagna LC, Koella JC: Malaria infection increases attractiveness of humans to mosquitoes. PLoS Biol 2005, 3:e298. 
69. Kiszewski A, Mellinger A, Spielman A, Malaney P, Sachs SE, Sachs J: A global index representing the stability of malaria transmission. Am J Trop Med Hyg 2004, 70:486-498.

doi:10.1186/1475-2875-10-303

Cite this article as: Eckhoff: A malaria transmission-directed model of mosquito life cycle and ecology. Malaria Journal 2011 10:303.

Submit your next manuscript to BioMed Central and take full advantage of:

- Convenient online submission

- Thorough peer review

- No space constraints or color figure charges

- Immediate publication on acceptance

- Inclusion in PubMed, CAS, Scopus and Google Scholar

- Research which is freely available for redistribution

Submit your manuscript at www.biomedcentral.com/submit

() BioMed Central 